\title{
First detection of ozone in the mid-infrared at Mars: implications for methane detection
}

\author{
K. S. Olsen ${ }^{1,2}$, F. Lefèvre ${ }^{2}$, F. Montmessin ${ }^{2}$, A. Trokhimovskiy ${ }^{3}$, L. Baggio ${ }^{2}$, A. Fedorova ${ }^{3}$, J. Alday ${ }^{1}$, \\ A. Lomakin ${ }^{3,4}$, D. A. Belyaev ${ }^{3}$, A. Patrakeev ${ }^{3}$, A. Shakun ${ }^{3}$, and O. Korablev ${ }^{3}$ \\ ${ }^{1}$ Department of Physics, University of Oxford, Oxford, UK \\ e-mail: Kevin.0lsen@physics.ox.ac.uk \\ ${ }^{2}$ Laboratoire Atmosphères, Milieux, Observations Spatiales (LATMOS/CNRS), Paris, France \\ ${ }^{3}$ Space Research Institute (IKI), Moscow, Russia \\ ${ }^{4}$ Moscow Institute of Physics and Technology, Moscow, Russian Federation
}

Received 8 April 2020 / Accepted 22 May 2020

\begin{abstract}
Aims. The ExoMars Trace Gas Orbiter was sent to Mars in March 2016 to search for trace gases diagnostic of active geological or biogenic processes.

Methods. We report the first observation of the spectral features of Martian ozone $\left(\mathrm{O}_{3}\right)$ in the mid-infrared range using the Atmospheric Chemistry Suite Mid-InfaRed (MIR) channel, a cross-dispersion spectrometer operating in solar occultation mode with the finest spectral resolution of any remote sensing mission to Mars.

Results. Observations of ozone were made at high northern latitudes $\left(>65^{\circ} \mathrm{N}\right)$ prior to the onset of the 2018 global dust storm $\left(\mathrm{L}_{\mathrm{s}}=163-193^{\circ}\right)$. During this fast transition phase between summer and winter ozone distribution, the $\mathrm{O}_{3}$ volume mixing ratio observed is 100-200 ppbv near $20 \mathrm{~km}$. These amounts are consistent with past observations made at the edge of the southern polar vortex in the ultraviolet range. The observed spectral signature of ozone at 3000-3060 $\mathrm{cm}^{-1}$ directly overlaps with the spectral range of the methane $\left(\mathrm{CH}_{4}\right) v_{3}$ vibration-rotation band, and it, along with a newly discovered $\mathrm{CO}_{2}$ band in the same region, may interfere with measurements of methane abundance.
\end{abstract}

Key words. planets and satellites: atmospheres - planets and satellites: composition - planets and satellites: detection planets and satellites: terrestrial planets - radiative transfer

\section{Introduction}

Ozone $\left(\mathrm{O}_{3}\right)$ on Mars was first observed by the Ultraviolet Spectrometers on Mariner 7 and 9 (Barth et al. 1973; Barth \& Hord 1971), which showed large variability and established seasonal trends. Since then, $\mathrm{O}_{3}$ has been observed by ground-based campaigns and spacecraft missions, but largely using absorption and emission features in the ultraviolet spectral range (e.g. Clancy et al. 2016; Perrier et al. 2006) or the thermal spectral range using the $9.7 \mu \mathrm{m}$ band (Espenak et al. 1991; Fast et al. 2006). Here, we report the first observations of ozone absorption in the midinfrared spectral region, between 3015 and $3050 \mathrm{~cm}^{-1}$, using the mid-infrared channel of the Atmospheric Chemistry Suite (ACS) Mid-InfaRed (MIR) onboard the ExoMars Trace Gas Orbiter (TGO). This spectral region is shared by the $v_{3}$ vibration-rotation band of methane $\left(\mathrm{CH}_{4}\right)$, as well as a newly discovered transition of $\mathrm{CO}_{2}$ (Trokhimovskiy et al. 2020). The ability to simultaneously resolve these species has an impact on current and past attempts to measure the abundance of methane in the atmosphere of Mars.

ACS MIR is a novel cross-dispersion spectrometer making solar occultation observations of the limb of the Martian atmosphere. It has the finest spectral resolution of any Martian remote-sensing instrument to date, and the solar occultation technique provides a high signal-to-noise ratio $(\mathrm{S} / \mathrm{N})$, and strong sensitivity to the vertical structure of the atmosphere. These characteristics were instrumental in observing, for the first time, the mid-infrared $003 \leftarrow 000$ transitions of $\mathrm{O}_{3}$ in the atmosphere of Mars.
Here we present solar occultation observations made in Mars year (MY) 34 by ACS MIR between solar longitude $\left(\mathrm{L}_{\mathrm{s}}\right) 163-193^{\circ}$ and north of $60^{\circ} \mathrm{N}$ (May-June 2018). In this region and time period, corresponding to the northern autumn equinox, we were able to observe significant amounts of ozone in the mid-infrared at altitudes below $30 \mathrm{~km}$. In the following section, we describe the TGO mission, ACS instrument, and spectral fitting method. In Sect. 3 we present our observations, analysis, and comparison to model results and the literature. Section 4 discusses the implications that this observation has for $\mathrm{CH}_{4}$, which is sought in the same wavenumber range of the infrared.

Ozone chemistry. The stability of the $\mathrm{CO}_{2}$ atmosphere on Mars depends on the abundances of odd hydrogen $\left(\mathrm{H}, \mathrm{OH}, \mathrm{HO}_{2}\right)$, which is a product of $\mathrm{H}_{2} \mathrm{O}$ photolysis. The primary source of $\mathrm{O}_{3}$ is a three-body reaction between $\mathrm{O}_{2}$ and $\mathrm{O}$, while the primary loss mechanism is the inverse reaction via photolysis. Such a cycle is neutral in terms of the quantity of odd oxygen remaining because $\mathrm{O}$ is converted to $\mathrm{O}_{3}$ and vice versa. The main net-loss pathway of the odd oxygen family $\mathrm{O}_{\mathrm{x}}=\mathrm{O}+\mathrm{O}_{3}$ is the reaction with $\mathrm{HO}_{2}\left(\mathrm{HO}_{2}+\mathrm{O} \rightarrow \mathrm{OH}+\mathrm{O}_{2}\right)$, which reduces $\mathrm{O}_{\mathrm{x}}$. Ozone is therefore a valuable tracer of the odd hydrogen chemistry that stabilises the chemical composition of Mars' atmosphere, as $\mathrm{H}$, $\mathrm{OH}$, or $\mathrm{HO}_{2}$ have never been directly measured. Since odd hydrogen is primarily produced by $\mathrm{H}_{2} \mathrm{O}$ photolysis, ozone is expected to be anti-correlated with water vapour (e.g. Clancy et al. 1996; Lefèvre \& Krasnopolsky 2017; Perrier et al. 2006, and references therein). 
The ozone profiles described here were all obtained at high northern latitudes around autumn equinox $\left(\mathrm{L}_{\mathrm{s}}=160-190^{\circ}\right)$. At these latitudes and during this period, ultraviolet measurements performed in nadir geometry show ozone columns that rapidly increase with time (Clancy et al. 2016; Perrier et al. 2006). This dramatic rise in ozone occurs in conjunction with the buildup of the winter polar vortex and the quick decline in water vapour and $\mathrm{O}_{3}$-destroying odd hydrogen. Observations of the ozone profile are sparse in the literature and do not cover the latitudes and local times sampled here. Indeed, almost all published profiles of ozone at high latitudes were measured later in the season, in the polar night (Gröller et al. 2018; Montmessin \& Lefèvre 2013; Perrier et al. 2006). The only exceptions are the four polar ozone profiles measured by solar occultation in the ultraviolet range by Piccialli et al. (2019), but those were obtained in the southern hemisphere and at the edge of a fully developed polar vortex $\left(\mathrm{L}_{\mathrm{s}} 56-68^{\circ}\right)$. Our knowledge of the vertical distribution of ozone is therefore still very limited, in particular in twilight conditions. However, all previous studies show that the largest ozone densities on Mars are always found in the polar vortices and that the polar ozone layer is usually located low in the atmosphere, typically between the surface and $20-25 \mathrm{~km}$ altitude.

\section{Methods}

The TGO was launched in 2016, began its nominal science phase in April 2018, and has completed 2 yr of observations at the time of publication. The primary scientific objectives of TGO are to detect any trace gases diagnostic of active geologic or biogenic activity, characterise and attempt to locate the possible sources of such trace gases, and characterise the water cycle on Mars (Vago et al. 2015). To achieve these goals, the TGO carries two suites of multi-channel spectrometers: ACS (Korablev et al. 2018), and the Nadir and Occultation for Mars Discovery (NOMAD; Vandaele et al. 2018). Both spectrometer suites have three channels and are capable of making observations in nadir, limb, and solar occultation viewing geometries.

The MIR channel of ACS is a cross-dispersion spectrometer consisting of a primary echelle grating, and a secondary grating used to separate diffraction orders (Korablev et al. 2018). The secondary grating rotates through several positions to access different simultaneous spectral ranges. This work uses position 12 , which covers the spectral range $2850-3250 \mathrm{~cm}^{-1}$ that contains the main $\mathrm{CH}_{4}$ absorption band. This channel has the finest spectral resolution $\left(0.043-0.047 \mathrm{~cm}^{-1}\right)$ of any atmospheric remote sensing Mars mission and operates solely in solar occultation mode, benefiting from high signal strength, long optical path length, and the ability to directly probe vertical structure.

Calibration for these observations was performed at Russia's Space Research Institute (IKI) and involves performing several corrections to the data before subtracting a dark signal from observations taken over a series of tangent altitudes and a solar reference measured above the top of the atmosphere. Transmission spectra are obtained by dividing the corrected absorption spectra by the solar reference. Prior to transmission calculation, corrections applied to the data include removing dead or saturated pixels, accounting for subpixel drifts over time, and an ortho-rectification procedure needed to extract one-dimensional spectra from the two-dimensional detector array. See Fedorova et al. (2020), Olsen et al. (2020), Trokhimovskiy et al. (2020) for more details.

The instantaneous field of view (IFOV) of the instrument optics observing the edge of the solar disc is on the order of 1-4 km when projected at the Martian limb. The Sun edge is imaged onto the detector array over approximately 15 rows, and from each row, a spectrum can be extracted. We use the intensity curve, which is a column of the detector array image and covers the vertical fields of view of each diffraction order (see Olsen et al. 2020), to identify a spectrum near the slit edge, which is closest to the centre of the solar disc.

Wavenumber calibration of each diffraction order is performed in two steps. An initial guess of wavenumber positions for the solar reference spectrum is compared to the solar spectrum measured by the Atmospheric Chemistry Experiment Fourier Transform Spectrometer (ACE-FTS; Hase et al. 2010) in order to obtain a calibrated wavenumber vector. This is then refined using an appropriately clear transmission observation (avoiding signal attenuation due to aerosols) using transmission lines from $\mathrm{CO}_{2}, \mathrm{CO}$, or $\mathrm{H}_{2} \mathrm{O}$, if they are available and of sufficient strength.

Spectral fitting is performed by the Gas Fitting software suite (GFIT or GGG) maintained by NASA's Jet Propulsion Laboratory (e.g. Irion et al. 2002; Wunch et al. 2011). Over a given fitting window, volume absorption coefficients are computed for each gas and a spectrum is computed line-by-line. Non-linear Levenberg-Marquardt minimisation is done to determine a bestfit spectrum by modifying volume mixing ratio (VMR) scaling factors for a set of target gases. A set of estimated slant column abundances for all observed tangent altitudes is inverted with calculated slant column paths traced through the atmosphere using a linear equation solver to obtain a retrieved VMR vertical profile. Volume absorption coefficients are computed using the HITRAN 2016 line list (Gordon et al. 2017; Olsen et al. 2019), supplemented by $\mathrm{H}_{2} \mathrm{O}$ broadening parameters for a $\mathrm{CO}_{2}$-rich atmosphere (Devi et al. 2017; Gamache et al. 2016).

The width of an instrument line shape is wavenumber dependent. Therefore, to obtain more accurate fits we use narrow fitting windows. The $\mathrm{S} / \mathrm{N}$ of each spectrum, corresponding to a diffraction order, is also highest in its centre due to the blaze function of the echelle grating. To avoid errors in spectral calibration, we avoid working toward the edges of the spectra and cover the centre of each order with two or three windows that are 5$6 \mathrm{~cm}^{-1}$ in width (a diffraction order in position 12 has a width of $19-21 \mathrm{~cm}^{-1}$ ). There remains a curvature in the baseline of the spectra. We use normalised spectra here and use an alpha shape, which finds the geometric area containing a spectrum, to estimate the baseline shape (Xu et al. 2019).

Accurate knowledge of temperature and pressure are critical for computing the number density of the atmosphere and performing accurate trace gas retrievals. In this work, temperature and pressure have been retrieved from observations of $\mathrm{CO}_{2}$ lines using coincident measurement made with the ACS near-infrared channel (Fedorova et al. 2020).

\section{Observations and results}

We first discovered the transmission signature of $\mathrm{O}_{3}$ in ACS MIR data during orbit 2476 (occultation N1), which was recorded on June 132018 , or $\mathrm{L}_{\mathrm{s}}=192.7^{\circ}$. The local time was $17: 16$, and the latitude and longitude of the tangent point were $66.5^{\circ} \mathrm{N}, 13.5^{\circ} \mathrm{E}$. This was shortly after the onset of the global dust storm of MY 34, which began around $\mathrm{L}_{\mathrm{s}}=190^{\circ}$ (Montabone et al. 2020), but at such a high latitude that its impact was not yet felt and observations with suitable transmittance were made down to $2.3 \mathrm{~km}$ above the aeroid. The signature was initially identified in order 182 , covering the spectral range $3047.5-3067.5 \mathrm{~cm}^{-1}$, shown in Fig. 1, with a computed best-fit spectrum. Once identified, the signature was also clearly identified in orders 180 and 181, 
K. S. Olsen et al.: First detection of ozone in the mid-infrared at Mars: implications for methane detection

a)

b)
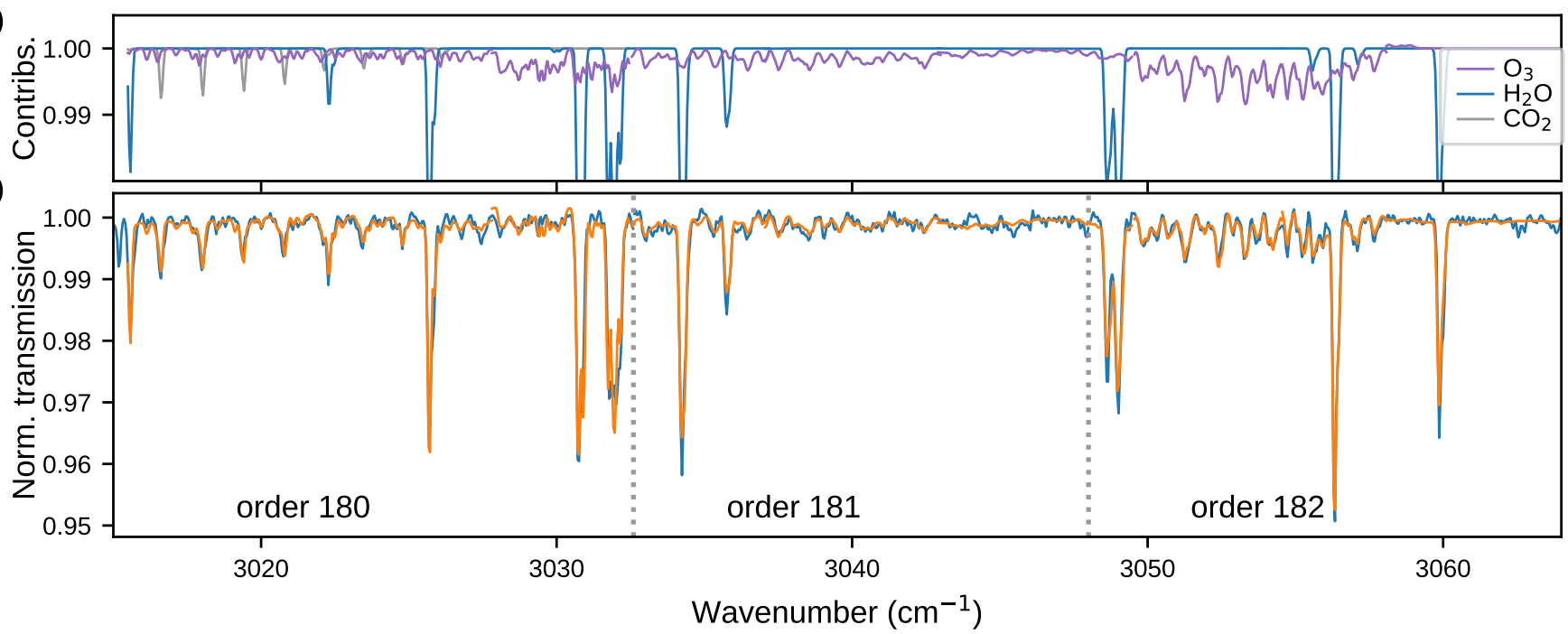

Fig. 1. Spectra recorded by ACS MIR at $5.5 \mathrm{~km}$ using secondary grating position 12 during occultation $2476 \mathrm{~N} 1$ on $\mathrm{L}_{\mathrm{s}}=192.7^{\circ}$ : (a) contributions from $\mathrm{O}_{3}, \mathrm{H}_{2} \mathrm{O}$, and $\mathrm{CO}_{2}$ to the best-fit for orders 180-182; (b) data and best-fits for orders 180-182.

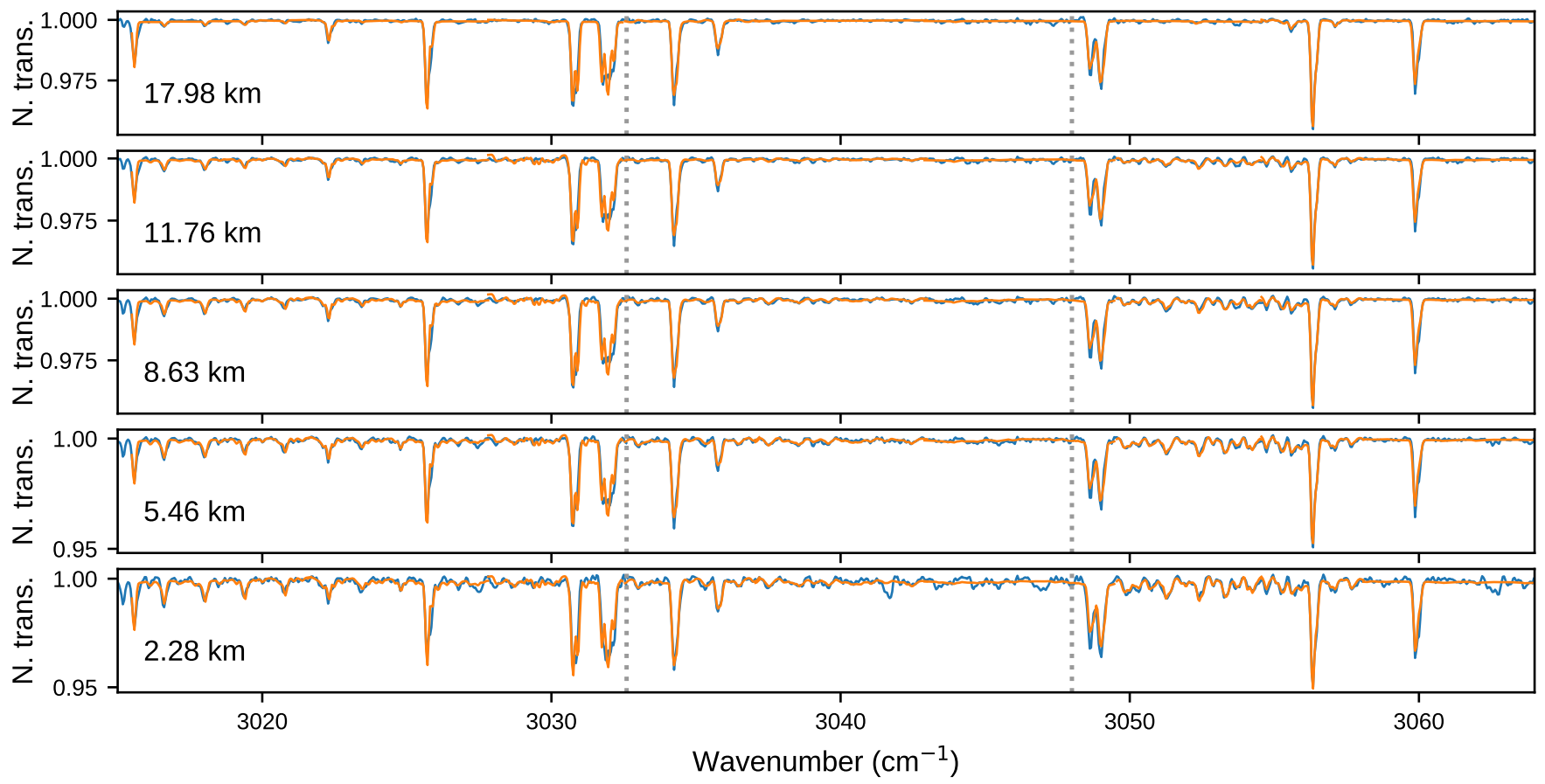

Fig. 2. Same as Fig. 1b, except at a range of altitudes.

shown in Fig. 1b. The signature is very small relative to nearby $\mathrm{H}_{2} \mathrm{O}$ absorption lines and is only apparent in a small number of observations, and at the lowest altitude levels. It is consistently visible over a range of observed tangent altitudes, and its evolution over altitude is also clearly distinguishable. Spectra from five consecutive tangent altitudes from the same occultation are shown in Fig. 2, from the lowest observable tangent altitude of $2.3 \mathrm{~km}$ (which had a transmission level of $30 \%$ ), up to $18 \mathrm{~km}$, above which the signature vanishes in the instrument noise (we note there was a corrupt observation near $15 \mathrm{~km}$ ). The $\mathrm{CO}_{2}$ lines towards the left side of order 180 in Figs. 1a, b are not included in any current line lists and have been identified as a magnetic dipole absorption band of the 628 isotopologue of $\mathrm{CO}_{2}$; they are reported in a separate paper (Trokhimovskiy et al. 2020) and the fit shown in Fig. 1b uses line positions calculated in Trokhimovskiy et al. (2020), and line strengths and widths estimated from the nearby $v_{2}+v_{3}$ band of the ${ }^{16} \mathrm{O}^{12} \mathrm{C}^{18} \mathrm{O}$ isotopologue of $\mathrm{CO}_{2}$. The spectral window covering this area was not used to estimate the $\mathrm{O}_{3}$ abundance since the $\mathrm{O}_{3}$ transitions here are relatively weak and the $\mathrm{CO}_{2}$ spectroscopy is still imprecise.

After the initial discovery, a careful search of all processed position 12 observations was undertaken. The signature was found in several other occultations, and often at higher altitudes. Figure 3 shows best fits in order 182 for four other occultations at altitudes near $15 \mathrm{~km}$ where the signature is strong and clear.

Figure 4 indicates where $\mathrm{O}_{3}$ was identified over the evolution of the ACS MIR occultation latitudes with time $\left(\mathrm{L}_{\mathrm{s}}\right)$. All of these 
Orb. $1833 \mathrm{~N} 1,14.2 \mathrm{~km}$
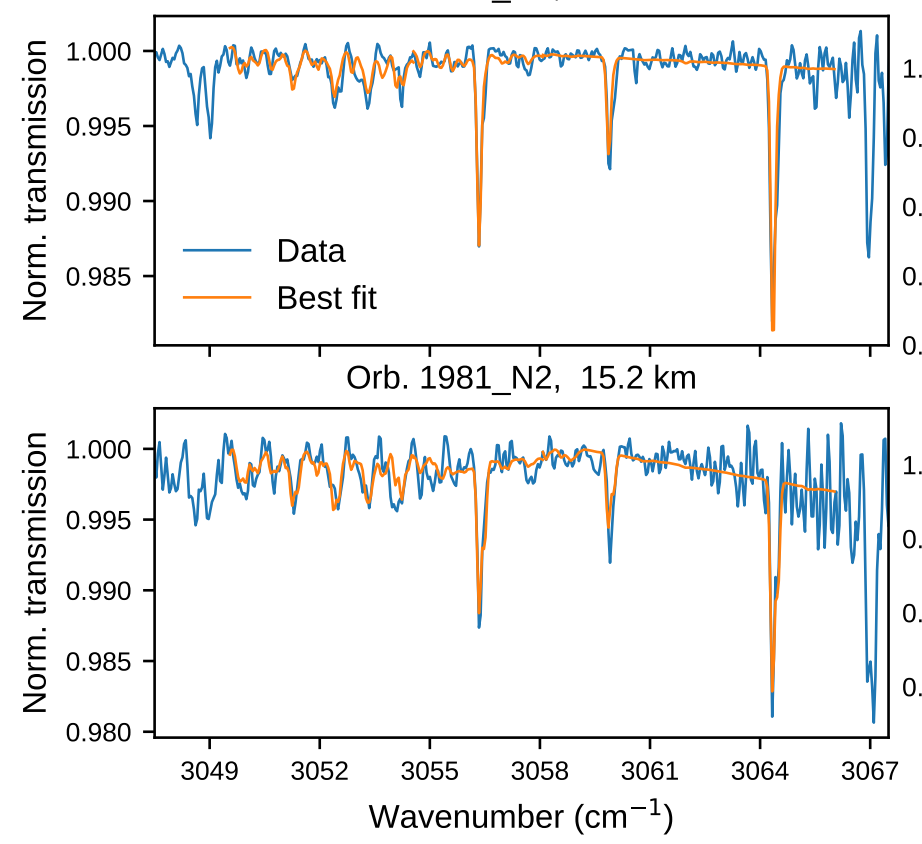

Orb. 1943_N2, 12.9 km

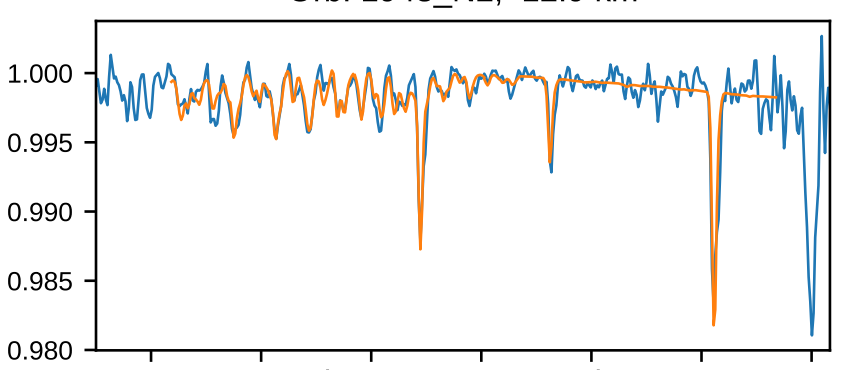

Orb. 2392_N1, $12.8 \mathrm{~km}$

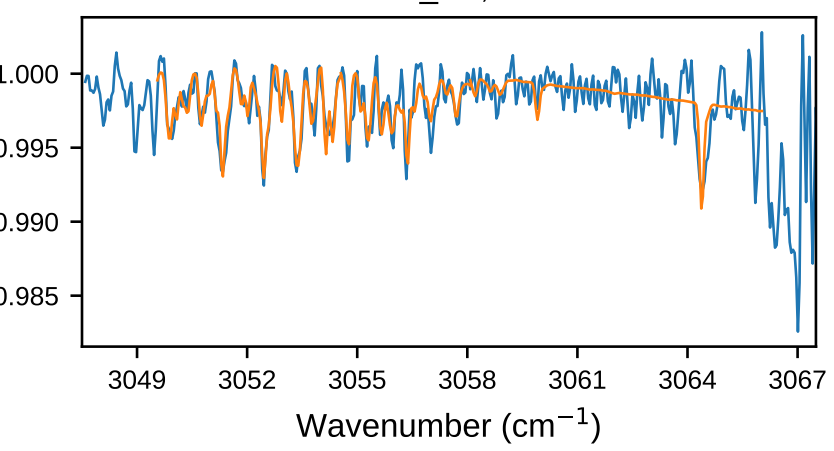

Fig. 3. Measured spectra and best-fits for order 182 for four occultations recorded between $L_{s}=160-200^{\circ}$ and north of $65^{\circ} \mathrm{N}$.

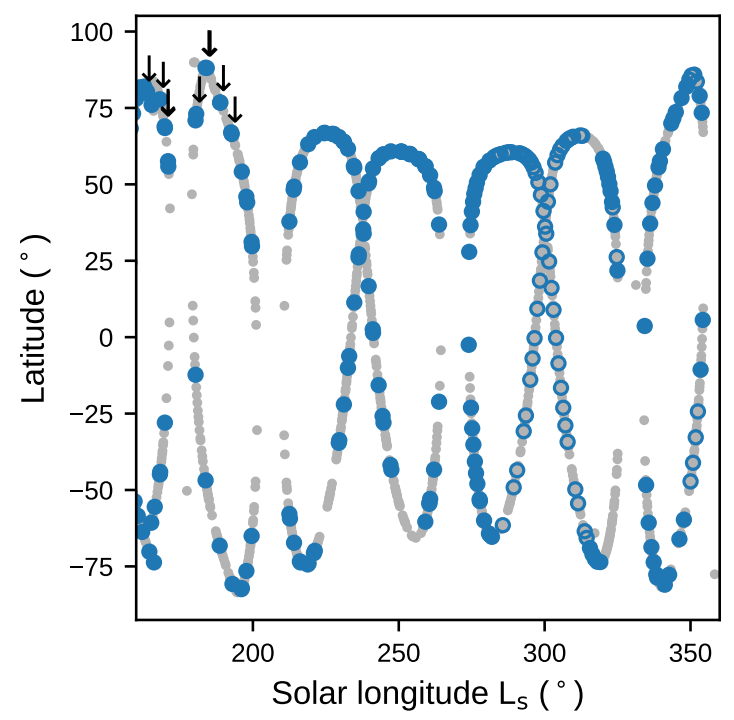

Fig. 4. Latitudes of ACS MIR occultation tangent points as a function of $\mathrm{L}_{\mathrm{s}}$. Dots in grey are ACS MIR occultations using grating positions other than 12. Filled blue circles are full-frame observations, and empty blue circles are partial-frame observations. Occultations exhibiting strong $\mathrm{O}_{3}$ absorption features are indicated with arrows.

observations occurred early in the mission, and at high northern latitudes, greater than $60^{\circ} \mathrm{N}$ and in the range $\mathrm{L}_{\mathrm{s}}=160-200^{\circ}$. This is consistent with previous studies of the climatology of Martian $\mathrm{O}_{3}$ that have reported accumulation over the poles during fall/winter (Clancy et al. 2016; Perrier et al. 2006), resulting from reduced destruction pathways caused by low water vapour content and solar insolation. However, according to these studies, the area of enhanced $\mathrm{O}_{3}$ should extend southward to $40^{\circ} \mathrm{N}$ and cover the time period $\mathrm{L}_{\mathrm{s}}=180-360^{\circ}$. We do not find convincing signatures of $\mathrm{O}_{3}$ over the northern extent of ACS MIR coverage in the range $\mathrm{L}_{\mathrm{s}}=200-280^{\circ}$ because of the onset of the global dust storm. Indeed, the dust storm generally sets an altitude limit on the vertical extent of solar occultation observations of $25-35 \mathrm{~km}$, below which meaningful signal is lost. In the range $L_{s}=280-380^{\circ}$, data volume constraints required us to download only partial detector frames (open circles in Fig. 4), which have not been fully processed.

Vertical profiles of retrieved number densities and mixing ratios are shown in Figs. 5a, b. The lower bound of the profiles indicates where the transmission signal dropped off to less than 0.05 . The upper altitude limit, denoted by the transition from a solid to dotted line, indicates where the $\mathrm{O}_{3}$ absorption features begin to be hidden in background noise, causing the retrieval uncertainty to grow larger than the VMR. The uncertainties shown in Fig. 5b are the sum of the partial derivatives computed for the inversion of the matrices containing the number densities and slant paths (Jacobian matrix). In the right panel, Fig. 5c, we also show vertical profiles generated by running the LMD general circulation model (GCM; Forget et al. 1999; Lefèvre et al. 2004) using a dust scenario for MY 34 (Montabone et al. 2020).

Except for the earliest profile measured at $\mathrm{L}_{\mathrm{s}}=163^{\circ}$, all profiles indicate that the $\mathrm{O}_{3}$ density strongly increases at lower altitudes. This suggests a gradual transition towards the surface $\mathrm{O}_{3}$ layer typically observed in the winter polar vortex. The largest $\mathrm{O}_{3}$ densities reach $10^{10}$ molec $\mathrm{cm}^{-3}$ at $10 \mathrm{~km}$ and below, in agreement with the polar profiles measured by the MEx SPectroscopie pour l'Investigation des Caractéristiques Atmosphériques de Mars (SPICAM) in the southern hemisphere (Lebonnois et al. 2006; Montmessin \& Lefèvre 2013).

SPICAM has also been used to measure the $\mathrm{O}_{3}$ mixing ratio near the south polar enhancement (Montmessin \& Lefèvre 2013; Piccialli et al. 2019). These latter authors observed strongly increasing $\mathrm{O}_{3}$ abundance below $30 \mathrm{~km}$ toward 100-300 ppbv, which was distinct from mid-latitude observations. These mixing ratios are comparable to those presented in Fig. 5b, which are between 100 and 300 ppbv.

In Fig. 5c, the LMD GCM ozone profiles co-located with the ACS measurements also show a large variability in the short period of $\mathrm{L}_{\mathrm{s}}$ sampled here. In general, the $\mathrm{O}_{3}$ mixing ratios 

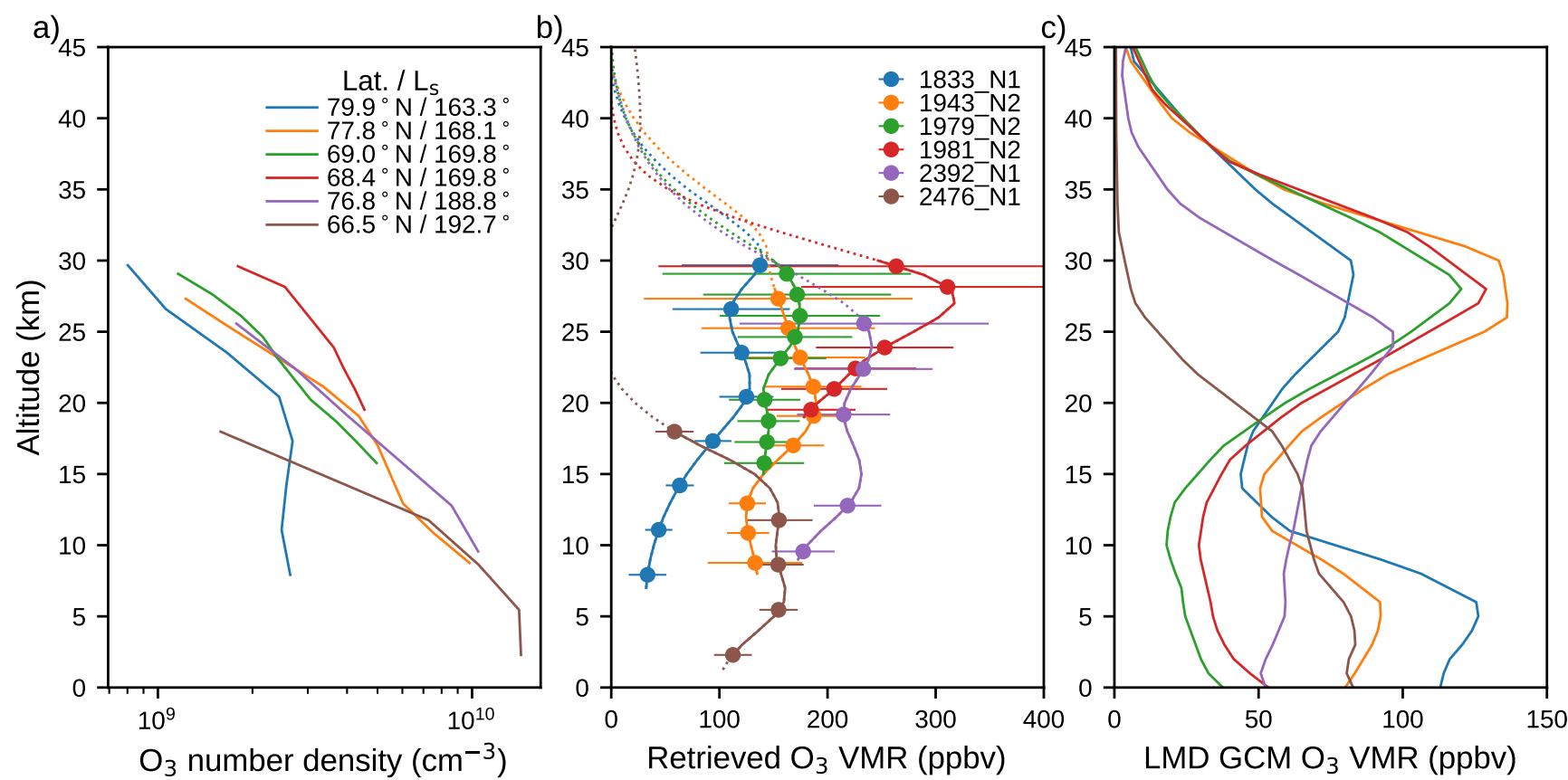

Fig. 5. Panel $a$ : retrieved number density or $\mathrm{O}_{3}$. Panel $b$ : retrieved VMR vertical profiles of $\mathrm{O}_{3}$. Panel $c$ : VMR vertical profiles of $\mathrm{O}_{3}$ extracted from the LMD GCM at corresponding $\mathrm{L}_{\mathrm{s}}$, locations, and local times. Colours indicating occultation number are shared between panels.

calculated by the model are underestimated by almost of factor of two relative to ACS MIR. This may reflect an imperfect timing in the model of the rapid decline in $\mathrm{H}_{2} \mathrm{O}$ that accompanies the buildup of the northern polar vortex at this time of the year. Below $20 \mathrm{~km}$, where ACS MIR observations are most sensitive, the model simulation shows a pronounced $\mathrm{O}_{3}$ minimum for the earlier profiles $\left(\mathrm{L}_{\mathrm{s}}=163-189^{\circ}\right)$ that is not seen in the measurements. The model outputs suggest that this problem is due to an overly strong poleward transport of $\mathrm{H}_{2} \mathrm{O}$-rich air originating from mid-latitudes. The observation presented in Fig. 5 in brown, $2476 \mathrm{~N} 1$, is an outlier in that ozone decreases with altitude above $10 \mathrm{~km}$. The other occultations from this period feature an increasing or roughly constant mixing ratio up to $30 \mathrm{~km}$. In the LMD GCM, the shape of the $\mathrm{O}_{3}$ profile for this latest observation $\left(\mathrm{L}_{\mathrm{s}}=192.7^{\circ}\right)$ is also characterised by a strong decrease above $20 \mathrm{~km}$ that contrasts with the earlier profiles. Examination of the model results shows that the change in the shape of this last $\mathrm{O}_{3}$ profile is related to a large increase in $\mathrm{H}_{2} \mathrm{O}$ above $20 \mathrm{~km}$, which is also observed at the same time and location by the ACS NIR instrument (Fedorova et al. 2020).

\section{Implications for $\mathrm{CH}_{4}$ observation}

Ozone absorption below $30 \mathrm{~km}$ in the mid-infrared range has important implications for searches for atmospheric methane. Past observations of methane in the atmosphere of Mars (Formisano et al. 2004; Krasnopolsky et al. 2004; Mumma et al. 2009; Webster et al. 2015) were a driving cause of the development of the ExoMars TGO mission. $\mathrm{CH}_{4}$ should have a relatively short lifetime in the atmosphere of Mars (several hundred years), meaning current observations require an active source (Lefèvre \& Forget 2009). A key objective of the TGO mission is to determine with certainty whether or not $\mathrm{CH}_{4}$ is present in the atmosphere of Mars and what its spatial and temporal variability is, and to localise any possible sources. This story continues to be intriguing as the first results from TGO reported an upper limit on the order of 50 pptv (Korablev et al. 2019), and ACS
MIR observations continue to reveal no methane after one MY. In its place, we have instead found the rare and previously undetected signatures of $\mathrm{O}_{3}$ and a new $\mathrm{CO}_{2}$ magnetic dipole band (Trokhimovskiy et al. 2020).

The strongest observed $\mathrm{O}_{3}$ features directly overlap important methane features used by the Planetary Fourier Spectrometer (PFS) on Mars Express, the Tunable Laser Spectrometer (TLS) on Curiosity, and ground-based observatories. Figure $6 \mathrm{a}$ compares the contributions to a transmission spectrum for $150 \mathrm{ppbv}$ of $\mathrm{O}_{3}$ and $1 \mathrm{ppbv}$ of methane. Figure $6 \mathrm{~b}$ shows a zoomed region surrounding the $\mathrm{CH}_{4} Q$-branch, and Fig. $6 \mathrm{c}$ shows a zoomed region surrounding the strongest line of the $\mathrm{CH}_{4}$ $R$-branch. Panels a and b also show 50-150 pptv of methane, approaching the upper limits reported by ACS (Korablev et al. 2019). In both cases, there is direct overlap between absorption lines of $\mathrm{CH}_{4}$ and $\mathrm{O}_{3}$, with absorption lines produced by $150 \mathrm{ppbv}$ $\mathrm{O}_{3}$ being deeper than those from 150 pptv of $\mathrm{CH}_{4}$. Reported values of methane abundance range from the background measured by TLS of 0.4 ppbv (Webster et al. 2018) to enhancements measured by TLS and PFS of 6-16 ppbv (Giuranna et al. 2019; Webster et al. 2015). We computed model spectra at the resolutions of both instruments and are confident that errors can be made when $\mathrm{CO}_{2}$ and $\mathrm{O}_{3}$ spectroscopy is not accounted for.

The $Q$-branch is especially important for spectrometers with coarser spectral resolution than ACS MIR, as the integrated $\mathrm{CH} 4$ lines that make up the $Q$-branch would have a higher magnitude than the individual lines in the $P$ - and $R$-branches. It would be observed in order 180 of ACS MIR, shown in Figs. 1 and 2, and is located in the same spectral range in which we report previously unknown $\mathrm{CO}_{2}$ lines (Trokhimovskiy et al. 2020), identified in Fig. 1a. The $Q$-branch is used by the PFS team in their $\mathrm{CH}_{4}$ analysis (Formisano et al. 2004; Giuranna et al. 2019), but with their spectral resolution, $1.3 \mathrm{~cm}^{-1}$, the entire $Q$-branch region is integrated into only two or three spectral points.

The $R$-branch consists of a series of broadly spaced lines, the strongest of which lies at $3057.7 \mathrm{~cm}^{-1}$. This line has been used exclusively by TLS, which has sufficiently fine spectral 
a)
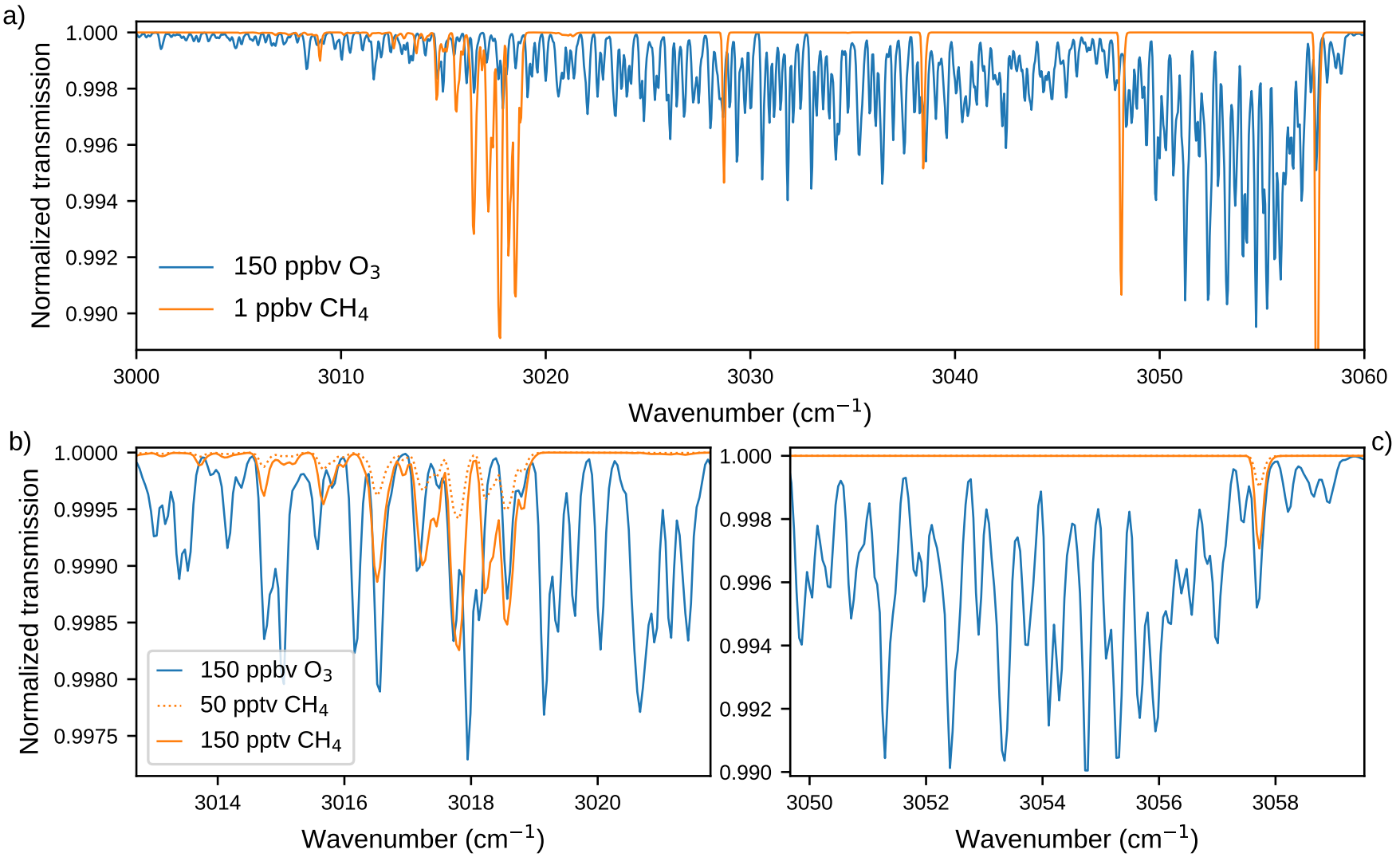

Fig. 6. Panel $a$ : modelled transmission spectrum contributions from $150 \mathrm{ppbv}$ of $\mathrm{O}_{3}$ and $1 \mathrm{ppbv}$ of $\mathrm{CH}_{4}$. Panel $b$ : close look at the modelled contributions around the $\mathrm{CH}_{4} Q$-branch, but using 50 and $150 \mathrm{pptv}$ of $\mathrm{CH}_{4}$. Panel $c$ : close look at the modelled contributions around the strongest line in the $\mathrm{CH}_{4} R$-branch.

resolution to observe the line as a triplet (Webster et al. 2015, 2018). The TLS instrument also has the capability to resolve the overlapping $\mathrm{O}_{3}$ line as a triplet. At this resolution, one pair of $\mathrm{O}_{3}$ and $\mathrm{CH}_{4}$ triplet lines directly overlap $\left(3057.685 \mathrm{~cm}^{-1}\right)$, and the other two pairs partially overlap. Despite this, the TLS team has not yet reported the abundance of ozone in Gale crater.

$\mathrm{CO}_{2}$ and $\mathrm{O}_{3}$ alone cannot account for the detections made by both teams. In the case of PFS, the previously unknown $\mathrm{CO}_{2}$ features would impact all observations equally, as $\mathrm{CO}_{2}$ is always present and well-mixed. The PFS team has instead identified $\mathrm{CH}_{4}$ in only a small number of observations (Formisano et al. 2004; Giuranna et al. 2019). Furthermore, we computed spectra with $\mathrm{O}_{3}$ at two and three times the quantities in our observations, and the sheer magnitude of $\mathrm{CH}_{4}$ observed by these latter authors (15 ppbv) is far too large to be easily mistaken for $\mathrm{O}_{3}$.

In the case of TLS, which takes measurements of $\mathrm{CH}_{4}$ at the surface and mostly at night where and when the $\mathrm{O}_{3}$ abundance is greatest, again, it is unlikely that the large quantity of $\mathrm{CH}_{4}$ observed (up to 9 ppbv) resulted from $\mathrm{O}_{3}$, yet the latter may interfere in the measurement of the background level of methane in the so-called enriched mode as both ozone and methane should sustain the same enrichment.

For ground-based observations, strong $\mathrm{O}_{3}$ absorption features from Earth's atmosphere must first be removed before retrieving mixing ratios for Mars (Krasnopolsky 2012; Mumma et al. 2009); $\mathrm{O}_{3}$ must be accounted for, although this step makes the retrieval more difficult (Zahnle et al. 2011). Finally, in the case of all previous observations, the rapid evolution and disappearance of $\mathrm{CH}_{4}$ are still not explained, although ozone chemistry is very rapid, with a lifetime on the order of days.

\section{Conclusion}

The faint spectral signature of ozone, an established trace gas in the Martian atmosphere, has been observed for the first time in the MIR spectral region by the ACS MIR instrument on ExoMars TGO. These observations are limited to high northern latitudes $\left(>65^{\circ} \mathrm{N}\right)$ and prior to the onset of the 2018 Mars global dust storm. During this time period, ACS MIR measurements provide new insight into the vertical structure of ozone around the northern fall equinox and show the variability its VMR can have shortly before the polar vortex is established. We observe the distinct presence of ozone with 100-200 ppbv at $20 \mathrm{~km}$ and below, which is close to the amounts measured in comparable conditions at the edge of the (southern) polar vortex. In general, the $\mathrm{O}_{3}$ mixing ratios retrieved by ACS MIR are higher than those calculated by the LMD GCM. This seems to result from an overly wet atmosphere in the model at the time (equinox) and location (high northern latitudes) sampled here; these parameters will need to be confirmed in the future by simultaneous measurements of water vapour by ACS NIR.

We look forward to the processing of observations made in MY 35 and southern winter. Dust obscured the northern ACS observations after $L_{\mathrm{s}} 190^{\circ}$, but observations made after $\mathrm{L}_{\mathrm{s}} \sim 30^{\circ}$ in the next MY are very clear, and we expect to be able to detect low-altitude ozone near the southern hemisphere.

The observation of this trace gas at higher-than-predicted VMRs below $30 \mathrm{~km}$ has important implications for the detection of methane in the atmosphere of Mars. This band, as well as the previously unidentified $\mathrm{CO}_{2}$ band, overlap and interfere with the $\mathrm{CH}_{4} v_{3}$ band used by TGO, MEx, and MSL to search 
for methane. Accounting for these absorption features improves our own spectral fitting and will lead to more accurate lower limits in the future. In conclusion, our study shows that the $\mathrm{O}_{3}$ lines we report here interfere with measurements of Martian methane, but a detailed reanalysis of these measurements is required to precisely assess their impact.

Acknowledgements. The ACS investigation was developed by the Space Research Institute (IKI) in Moscow, and the Laboratoire Atmosphères, Milieux, Observations Spatiales (LATMOS/CNRS) in Paris. The investigation was funded by Roscosmos, the National Centre for Space Studies of France (CNES) and the Ministry of Science and Education of Russia. The GGG software suite is maintained at JPL (tccon-wiki.caltech.edu). This work was funded by the Natural Sciences and Engineering Research Council of Canada (NSERC) (PDF-5168952018), the UK Space Agency (ST/T002069/1) and the National Centre for Space Studies of France (CNES). All spectral fitting was performed by KSO using the GGG software suite. The interpretation of the results was done by K.S.O. and F.L. The processing of ACS spectra is done at IKI by A.T. and at LATMOS by L.B. Input and aid on spectral fitting were given by J.A., D.B., A.F., A.L., and

F.M. The ACS instrument was designed, developed, and operated by A.P., A.S., A.T., F.M., and O.K.

\section{References}

Barth, C. A., \& Hord, C. W. 1971, Science, 173, 197

Barth, C. A., Hord, C. W., Stewart, A. I., et al. 1973, Science, 179, 795

Clancy, R. T., Wolff, M. J., James, P. B., et al. 1996, J. Geophys. Res., 101, 12777

Clancy, T. R., Wolff, M. J., Lefèvre, F., et al. 2016, Icarus, 266, 112

Devi, V. M., Benner, D. C., Sung, K., et al. 2017, J. Quant. Spectr. Rad. Transf., 203, 158

Espenak, F., Mumma, M. J., Kostiuk, T., \& Zipoy, D. 1991, Icarus, 92, 252

Fast, K., Kostiuk, T., Espenak, F., et al. 2006, Icarus, 181, 419

Fedorova, A. A., Montmessin, F., Korablev, O., et al. 2020, Science, 367, 297

Forget, F., Hourdin, F., Fournier, R., et al. 1999, J. Geophys. Res., 104, 24155

Formisano, V., Atreya, S., Encrenaz, T., Ignatiev, N., \& Giuranna, M. 2004, Science, 306, 1758

Gamache, R. R., Farese, M., \& Renaud, C. L. 2016, J. Mol. Spectr.., 326, 144

Giuranna, M., Viscardy, S., Daerden, F., et al. 2019, Nat. Geosci., 1752
Gordon, I. E., Rothman, L. S., Hill, C., et al. 2017, J. Quant. Spectr. Rad. Transf., 203, 3

Gröller, H., Montmessin, F., Yelle, R. V., et al. 2018, J. Geophys. Res., 123, 1449

Hase, F., Wallace, L., McLeod, S. D., Harrison, J. J., \& Bernath, P. F. 2010, J. Quant. Spectr. Rad. Transf., 111, 521

Irion, F. W., Gunson, M. R., Toon, G. C., et al. 2002, Appl. Opt., 41, 6968

Korablev, O., Montmessin, F., Trokhimovskiy, A., et al. 2018, Space Sci. Rev., 214, 7

Korablev, O., Vandaele, A. C., Montmessin, F., et al. 2019, Nature, 568, 517

Krasnopolsky, V. A. 2012, Icarus, 217, 144

Krasnopolsky, V. A., Maillard, J. P., \& Owen, T. C. 2004, Icarus, 172, 537

Lebonnois, S., Quémerais, E., Montmessin, F., et al. 2006, J. Geophys. Res., 111, E09S05

Lefèvre, F., \& Forget, F. 2009, Nature, 460, 720

Lefèvre, F., \& Krasnopolsky, V. 2017, in The Atmosphere and Climate of Mars, eds. R. M. Haberle, R. T. Clancy, F. Forget, M. D. Smith, \& R. W. Zurek, Cambridge Planetary Science (Cambridge: Cambridge University Press), 405

Lefèvre, F., Lebonnois, S., Montmessin, F., \& Forget, F. 2004, J. Geophys. Res., 109, E07004

Montabone, L., Spiga, A., Kass, D., et al. 2020, J. Geophys. Res., 125

Montmessin, F., \& Lefèvre, F. 2013, Nat. Geosci., 6, 930

Mumma, M. J., Villanueva, G. L., Novak, R. E., et al. 2009, Science, 323, 1041

Olsen, K. S., Boone, C. D., Toon, G. C., et al. 2019, J. Quant. Spectr. Rad. Transf., 236, 106590

Olsen, K. S., Lefèvre, F., Montmessin, F., et al. 2020, Nat. Geosci., submitted

Perrier, S., Bertaux, J. L., Lefèvre, F., et al. 2006, J. Geophys. Res., 111

Piccialli, A., Vandaele, A. C., Trompet, L., et al. 2019, Icarus, 113598

Trokhimovskiy, A., Perevalov, V., Korablev, O., et al. 2020, A\&A, 639, A142

Vago, J., Witasse, O., Svedhem, H., et al. 2015, Sol. Syst. Res., 49, 518

Vandaele, A. C., Lopez-Moreno, J.-J., Patel, M. R., et al. 2018, Space Sci. Rev., 214, 80

Webster, C. R., Mahaffy, P. R., Atreya, S. K., et al. 2015, Science, 347, 415

Webster, C. R., Mahaffy, P. R., Atreya, S. K., et al. 2018, Science, 360, 1093

Wunch, D., Toon, G. C., Blavier, J. L., et al. 2011, Phil. Trans. R. Soc. A, 369, 2087

Xu, X., Cisewski-Kehe, J., Davis, A. B., Fischer, D. A., \& Brewer, J. M. 2019 AJ, 157, 243

Zahnle, K., Freedman, R. S., \& Catling, D. C. 2011, Icarus, 212, 493 